\title{
Modeling inelastic phonon scattering in atomic- and molecular-wire junctions
}

\author{
Paulsson, Magnus; Frederiksen, Thomas; Brandbyge, Mads
}

Published in:

Physical Review B Condensed Matter

Link to article, DOI:

10.1103/PhysRevB.72.201101

Publication date:

2005

Document Version

Publisher's PDF, also known as Version of record

Link back to DTU Orbit

Citation (APA):

Paulsson, M., Frederiksen, T., \& Brandbyge, M. (2005). Modeling inelastic phonon scattering in atomic- and molecular-wire junctions. Physical Review B Condensed Matter, 72(20), 201101.

https://doi.org/10.1103/PhysRevB.72.201101

\section{General rights}

Copyright and moral rights for the publications made accessible in the public portal are retained by the authors and/or other copyright owners and it is a condition of accessing publications that users recognise and abide by the legal requirements associated with these rights.

- Users may download and print one copy of any publication from the public portal for the purpose of private study or research.

- You may not further distribute the material or use it for any profit-making activity or commercial gain

- You may freely distribute the URL identifying the publication in the public portal

If you believe that this document breaches copyright please contact us providing details, and we will remove access to the work immediately and investigate your claim 


\title{
Modeling inelastic phonon scattering in atomic- and molecular-wire junctions
}

\author{
Magnus Paulsson,* Thomas Frederiksen, and Mads Brandbyge \\ NanoDTU, MIC_Department of Micro and Nanotechnology, Technical University of Denmark, Ørsteds Plads, Building 345E, \\ DK-2800 Lyngby, Denmark
}

(Received 9 September 2005; published 28 November 2005)

\begin{abstract}
Computationally inexpensive approximations describing electron-phonon scattering in molecular-scale conductors are derived from the nonequilibrium Green's function method. The accuracy is demonstrated with a first-principles calculation on an atomic gold wire. Quantitative agreement between the full nonequilibrium Green's function calculation and the newly derived expressions is obtained while simplifying the computational burden by several orders of magnitude. In addition, analytical models provide intuitive understanding of the conductance including nonequilibrium heating and provide a convenient way of parameterizing the physics. This is exemplified by fitting the expressions to the experimentally observed conductances through both an atomic gold wire and a hydrogen molecule.
\end{abstract}

DOI: 10.1103/PhysRevB.72.201101

The rapid evolution in electronics towards smaller and faster devices will eventually reach the fundamental level set by the atomistic structure of matter. Atomic-size conductors take this development to the extreme of miniaturization, ${ }^{1}$ and understanding their properties is an important problem in the emerging fields of nanoelectronics and molecular electronics. One relevant aspect is the study of the effects caused by atomic vibrations, since inelastic scattering of traversing electrons and energy dissipation play essential roles for device characteristics, working conditions, and stability. Vibrational signals can also be used to extract information about the detailed microscopic configuration, which usually cannot be imaged simultaneously with a transport measurement. Inelastic effects have in the recent years been studied in a variety of nanoscale systems, e.g., single molecules on surfaces probed with the scanning tunneling microscope (STM) ${ }^{2}$ molecules in break junctions, ${ }^{3}$ and metallic atomic wires. ${ }^{4}$

Theoretical descriptions of inelastic transport through small devices connected to metallic contacts include the many-body theory in the Coulomb blockade regime, ${ }^{5}$ singleparticle first-order perturbation approaches, ${ }^{6,7}$ i.e., Fermi's golden rule (FGR), as well as calculations to infinite order based on the self-consistent Born approximation (SCBA) combined with nonequilibrium Green's functions (NEGF).${ }^{8-10}$ Our paper is based on the SCBA, which in contrast to FGR takes the many-particle nature of the problem into account. However, the SCBA method is computationally very demanding especially when used in combination with first-principles electronic structure methods. Moreover, the SCBA does not yield simple formulas that can be used to extract information from experimental data.

In this paper we develop methods that vastly simplify the SCBA approach. The main results are analytical formulas for the current and power derived from a lowest-order expansion (LOE) of the SCBA expressions. In particular, we show how first-principles SCBA calculations on atomic gold wires can be accurately described by the LOE with minimal computational effort. Moreover, we derive compact analytical expressions using two simple models. These latter models are able to fit both the theoretical SCBA results as well as experiments using the electron-hole damping rate of the phonon as the central parameter. ${ }^{11}$
PACS number(s): 73.23.-b, 73.63.-b, 72.10.Di

Phonon scattering is included in the SCBA method as self-energies to the electronic description. We use the undamped phonon Green's functions to express these selfenergies in the device subspace as ${ }^{12,13,19}$

$$
\begin{aligned}
\Sigma_{\mathrm{ph}}^{\lessgtr}(E)= & \sum_{\lambda} \mathbf{M}_{\lambda}\left[\left(n_{\lambda}+1\right) \mathbf{G}^{\lessgtr}\left(E \pm \hbar \omega_{\lambda}\right)\right. \\
& \left.+n_{\lambda} \mathbf{G}^{\lessgtr}\left(E \mp \hbar \omega_{\lambda}\right)\right] \mathbf{M}_{\lambda}, \\
\Sigma_{\mathrm{ph}}^{r}(E)= & \frac{1}{2}\left[\Sigma_{\mathrm{ph}}^{>}(E)-\Sigma_{\mathrm{ph}}^{<}(E)\right]-\frac{i}{2} \mathcal{H}\left\{\Sigma_{\mathrm{ph}}^{>}\left(E^{\prime}\right)-\Sigma_{\mathrm{ph}}^{<}\left(E^{\prime}\right)\right\}(E) .
\end{aligned}
$$

Here, $\mathbf{M}_{\lambda}$ is the electron-phonon coupling matrix for phonon mode $\lambda$ occupied by $n_{\lambda}$ phonons with energy $\hbar \omega_{\lambda}$. The lesser and greater self-energy matrices $\Sigma_{\mathrm{ph}}^{\lessgtr}$ are given by two terms corresponding to absorption (emission) of phonon quanta. We furthermore assume that these self-energies can be used in nonequilibrium with a bias-dependent phonon occupation number $n_{\lambda}(V)$. The retarded self-energy can then be obtained from the greater and lesser parts using the Hilbert transform $\left(\mathcal{H}\left\{f\left(E^{\prime}\right)\right\}(E)=1 / \pi \mathcal{P} \int f\left(E^{\prime}\right) /\left(E-E^{\prime}\right) d E^{\prime}\right)$.

The computational difficulty of solving the SCBA equations stems from the coupling of Green's functions in energy. Calculations usually involve a numerical energy grid that has to be fine enough to resolve the low temperature structure of the Fermi function, while at the same time span a large energy range to cover phonon energies, applied bias, and allow an accurate computation of the Hilbert transform that is nonlocal in energy. The current and power are then computed as integrals over this energy grid. ${ }^{9,12,13}$

These difficulties can be overcome if (i) the electronphonon coupling is weak, i.e., the probability for multiphonon processes is low, and (ii) the density of states (DOS) of the contacts and the device are slowly varying over a few phonon-energies around the Fermi energy $E_{F}$, i.e., in the notation used below, $\mathbf{G}^{r}(E) \approx \mathbf{G}^{r}\left(E_{F}\right)$ and $\boldsymbol{\Gamma}_{1,2}(E) \approx \boldsymbol{\Gamma}_{1,2}\left(E_{F}\right)$. These approximations are valid for systems where (i) the electron spends a short time compared to the phonon scattering time in the device and (ii) the closest resonance energy 
$\left(E_{\text {res }}\right)$ is either far away from the Fermi energy $\left(\left|E_{\text {res }}-E_{F}\right|\right.$ $\gg \Gamma, e V$, and $\hbar \omega)$ or the broadening by the contacts is large $\left(\Gamma \gg e V, \hbar \omega\right.$, and $\left.\left|E_{\text {res }}-E_{F}\right|\right)$. The expressions for the current and power ${ }^{9,12,13}$ can then be expanded to lowest order (second) in the electron-phonon coupling and the integration over energy performed analytically. The power dissipated into the phonon system $P^{\mathrm{LOE}}$ can, after lengthy derivations, be written

$$
\begin{aligned}
P^{\mathrm{LOE}}= & \sum_{\lambda} \frac{\left(\hbar \omega_{\lambda}\right)^{2}}{\pi \hbar}\left[n_{B}\left(\hbar \omega_{\lambda}\right)-n_{\lambda}\right] \operatorname{Tr}\left[\mathbf{M}_{\lambda} \mathbf{A} \mathbf{M}_{\lambda} \mathbf{A}\right] \\
& +\mathcal{P}\left(V, \hbar \omega_{\lambda}, T\right) \operatorname{Tr}\left[\mathbf{M}_{\lambda} \mathbf{G} \boldsymbol{\Gamma}_{1} \mathbf{G}^{\dagger} \mathbf{M}_{\lambda} \mathbf{G} \boldsymbol{\Gamma}_{2} \mathbf{G}^{\dagger}\right], \\
\mathcal{P}= & \frac{\hbar \omega}{\pi \hbar} \frac{\left[\cosh \left(\frac{e V}{k T}\right)-1\right] \operatorname{coth}\left(\frac{\hbar \omega}{2 k T}\right) \hbar \omega-e V \sinh \left(\frac{e V}{k T}\right)}{\cosh \left(\frac{\hbar \omega}{k T}\right)-\cosh \left(\frac{e V}{k T}\right)},
\end{aligned}
$$

where $n_{B}$ is the Bose-Einstein distribution, which appears naturally from the integration of the Fermi functions of the electrons in the contacts. Here, $\mathbf{G}=\mathbf{G}^{r}\left(E_{F}\right), \boldsymbol{\Gamma}_{1,2}=\boldsymbol{\Gamma}_{1,2}\left(E_{F}\right)$, and $\mathbf{A}=i\left(\mathbf{G}-\mathbf{G}^{\dagger}\right)$ are the noninteracting, i.e., without phonon interactions, retarded Green's function, the broadening by the contacts, and spectral function at $E_{F}$, respectively.

From Eq. (3) we see that the power can be decomposed into terms corresponding to the individual phonon modes. We also note that the first term describes the power balance between the electron and phonon systems (at zero bias) with an electron-hole damping rate $\gamma_{\mathrm{eh}}^{\lambda}=\omega_{\lambda} / \pi \operatorname{Tr}\left[\mathbf{M}_{\lambda} \mathbf{A} \mathbf{M}_{\lambda} \mathbf{A}\right]$ and is in fact equivalent to the FGR expression. ${ }^{11,14}$ The second term is even in bias and gives the phonon absorption (emission) at nonequilibrium; it is negligible at low bias $(\mathrm{eV}$ $\ll \hbar \omega)$, turns on at the phonon energy, and becomes linear in voltage at high bias $(e V \gg \hbar \omega)$.

Using the same approximations, the current through the device $I^{\mathrm{LOE}}$ is given by ${ }^{15}$

$$
\begin{aligned}
I^{\mathrm{LOE}}= & \frac{e^{2} V}{\pi \hbar} \operatorname{Tr}\left[\mathbf{G} \boldsymbol{\Gamma}_{2} \mathbf{G}^{\dagger} \boldsymbol{\Gamma}_{1}\right] \\
& +\sum_{\lambda} \mathcal{I}^{\operatorname{Sym}}\left(V, \hbar \omega_{\lambda}, T, n_{\lambda}\right) \operatorname{Tr}\left[\mathbf { G } ^ { \dagger } \boldsymbol { \Gamma } _ { 1 } \mathbf { G } \left\{\mathbf{M}_{\lambda} \mathbf{G} \boldsymbol{\Gamma}_{2} \mathbf{G}^{\dagger} \mathbf{M}_{\lambda}\right.\right. \\
& \left.\left.+\frac{i}{2}\left(\boldsymbol{\Gamma}_{2} \mathbf{G}^{\dagger} \mathbf{M}_{\lambda} \mathbf{A} \mathbf{M}_{\lambda}-\text { H.c. }\right)\right\}\right] \\
& +\sum_{\lambda} \mathcal{I}^{\mathrm{Asym}}\left(V, \hbar \omega_{\lambda}, T\right) \operatorname{Tr}\left[\mathbf { G } ^ { \dagger } \boldsymbol { \Gamma } _ { 1 } \mathbf { G } \left\{\boldsymbol{\Gamma}_{2} \mathbf{G}^{\dagger} \mathbf{M}_{\lambda} \mathbf{G}\left(\boldsymbol{\Gamma}_{2}-\boldsymbol{\Gamma}_{1}\right)\right.\right. \\
& \left.\left.\times \mathbf{G}^{\dagger} \mathbf{M}_{\lambda}+\text { H.c. }\right\}\right],
\end{aligned}
$$$$
\mathcal{I}^{\mathrm{Sym}}=\frac{e}{\pi \hbar}\left(2 e V n_{\lambda}+\frac{\hbar \omega_{\lambda}-e V}{e^{\left(\hbar \omega_{\lambda}-e V\right) / k T}-1}-\frac{\hbar \omega_{\lambda}+e V}{e^{\left(\hbar \omega_{\lambda}+e V\right) / k T}-1}\right),
$$$$
\mathcal{I}^{\text {Asym }}=\frac{e}{2 \pi \hbar} \int_{-\infty}^{\infty}\left[n_{F}(E)-n_{F}(E-e V)\right]
$$$$
\times \mathcal{H}\left\{n_{F}\left(E^{\prime}+\hbar \omega_{\lambda}\right)-n_{F}\left(E^{\prime}-\hbar \omega_{\lambda}\right)\right\}(E) d E,
$$

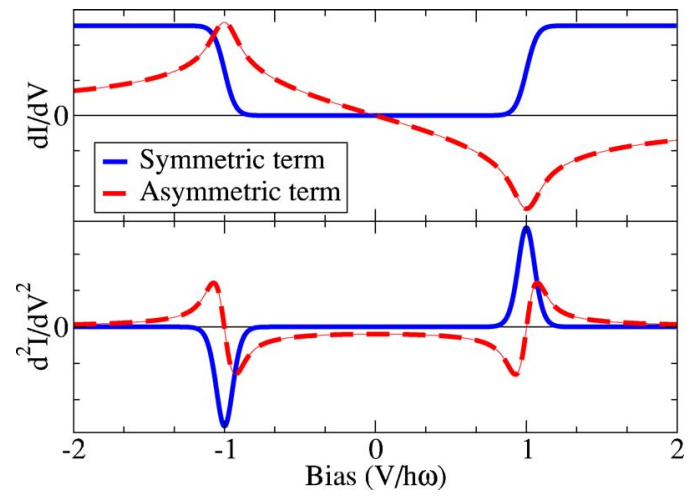

FIG. 1. (Color online) Universal functions [Eqs. (6) and (7)] giving the phonon contribution to the current. The differential conductance $d I / d V$ and the second derivative signals are shown for one phonon mode with the bias in units of the phonon energy at a temperature $k T=0.025 \hbar \omega$. For the symmetric term, the FWHM of the second derivative peak is approximately $5.4 k T$ (see Ref. 18).

where $n_{F}$ is the Fermi function, the bias is defined via $\mathrm{eV}$ $=\mu_{2}-\mu_{1}$, and the conductance quantum $G_{0}=e^{2} / \pi \hbar$ appears naturally. In contrast to the first Born approximation, these expressions are current conserving like SCBA.

The current expression retains the structure of the Landauer expression [the first term of Eq. (5)] and gives correction terms for each phonon mode. The phonon terms can in turn be divided into a "symmetric" term $\mathcal{I}^{\text {Sym }}$ where the differential conductance $d I / d V$ is even in bias, and an "asymmetric" term containing the Hilbert transform $\mathcal{I}^{\text {Asym }}$ yielding an odd contribution. Note the simple factorization into terms depending on the electronic structure at $E_{F}$ and universal functions $\mathcal{I}^{\text {Sym }}$ and $\mathcal{I}^{\text {Asym }}$ that yield the line shape of the inelastic signals in the $I-V$ (see Fig. 1). Whether the conductance increases or decreases due to phonon scattering depends on the sign of the traces in Eq. (5) and will be discussed further below. Examination of the "asymmetric" term in Eq. (5) shows that it is zero for symmetric systems. Although experimentally measured conductances contain asymmetric signals, the size of these signal is usually small in the published curves. At present it is unclear if they are caused by phonons or other effects.

As we have shown previously heating of the phonon system should be considered ${ }^{9}$ that makes the number of phonons $n_{\lambda}$ bias dependent. The simplest way to include nonequilibrium heating is to write down a rate equation, including an external damping rate $\gamma_{d}^{\lambda}$ of the phonons

$$
\dot{n}_{\lambda}=\frac{P_{\lambda}^{\mathrm{LOE}}}{\hbar \omega}+\gamma_{d}^{\lambda}\left[n_{B}\left(\hbar \omega_{\lambda}\right)-n_{\lambda}\right]
$$

where $P_{\lambda}^{\mathrm{LOE}}$ is the power dissipated into the individual phonon modes. ${ }^{20}$ The steady state occupation $n_{\lambda}$ is easily found. Substituting the result into Eqs. (5)-(7) gives a computationally simple but powerful formula for the current through the device including heating of the phonon system.

To judge the accuracy of the LOE approach, we compare the LOE results to the full SCBA solution for a four atom gold wire (see Fig. 2). The SCBA calculation was performed 


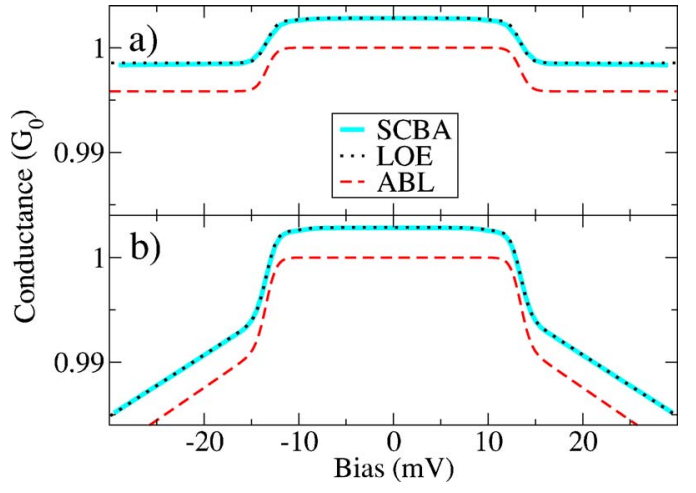

FIG. 2. (Color online) Comparison between the SCBA results and the LOE expressions [Eq. (5)] (a) without heating and (b) with heating $\left(\gamma_{d}=0\right)$ at $T=4.2 \mathrm{~K}$ for a 4-atom Au-wire. The parameters for the ABL model [Eq. (12)] were extracted directly from the DFT calculations, $\gamma_{\mathrm{eh}}=5.4 \times 10^{10} \mathrm{~s}^{-1}$ and $\hbar \omega=13.4 \mathrm{meV}$.

as described previously, ${ }^{9}$ where the Hamiltonian, phonon modes, and electron-phonon interaction were obtained from density functional calculations (DFT). The excellent agreement between the full SCBA and the LOE expression can be understood by noting that the DOS of a gold wire is slowly changing over an energy range much greater than the phonon energies. In addition, the electrons only spend a small time in the wire ${ }^{6}$ compared to the electron-hole damping rate. Importantly, the LOE conductance calculations were performed in less than a minute on a regular PC, compared to several hours for the SCBA calculations. The LOE approach thus opens up the possibility to study inelastic scattering with first-principles methods for large systems, e.g., organic molecules.

To gain further insight into the expressions presented above, we consider a single electronic site with symmetric contacts $\Gamma=\Gamma_{1}=\Gamma_{2}$ coupled to one phonon mode. Introducing the transmission probability $\tau=|G|^{2} \Gamma^{2}$ and the electronhole damping rate $\gamma_{\mathrm{eh}}=4(\omega / \pi) M^{2} \tau^{2} / \Gamma^{2}$, we obtain

$$
\begin{aligned}
& P_{\mathrm{one}}^{\mathrm{LOE}}=\gamma_{\mathrm{eh}} \hbar \omega\left[n_{B}(\hbar \omega)-n\right]+\frac{\gamma_{\mathrm{eh}}}{4} \frac{\pi \hbar}{\hbar \omega} \mathcal{P}, \\
& I_{\mathrm{one}}^{\mathrm{LOE}}=\frac{e^{2}}{\pi \hbar} \tau V+e \gamma_{\mathrm{eh}} \frac{1-2 \tau}{4} \frac{\pi \hbar}{e \hbar \omega} \mathcal{I}^{\mathrm{Sym}} .
\end{aligned}
$$

We note that, from the term $1-2 \tau$ in Eq. (10), the conductance will increase due to phonon scattering for low conductance systems $(\tau<1 / 2)$ and decrease for highly conducting systems $(\tau>1 / 2)$. The LOE approach directly provides the sign of the conductance change in contrast to FGR approaches where this requires careful considerations. ${ }^{6,7}$

The conductance through a single hydrogen molecule has been measured using a platinum break junction setup. ${ }^{3,16} \mathrm{Be}-$ cause the elastic current is carried through a single molecular orbital, ${ }^{16}$ the single level model fits the experiment very well [see Fig. 3(a)]. The best fit is obtained using a negligible external damping of the phonon mode $\left(\gamma_{d} \ll \gamma_{\mathrm{eh}}\right)$, which can be understood physically from the mass difference between the hydrogen molecule and the platinum atoms of the break

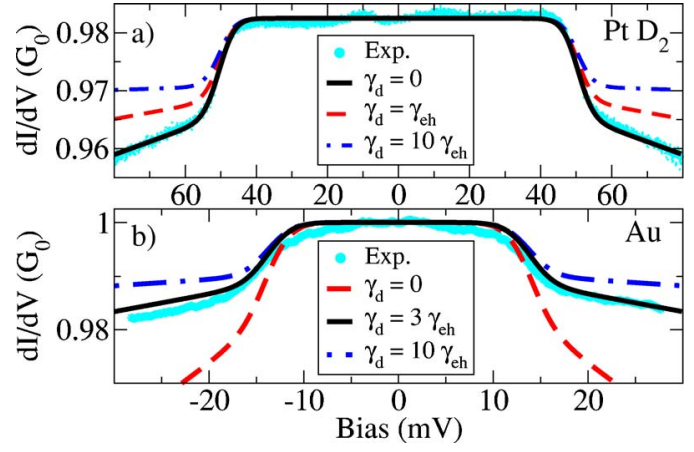

FIG. 3. (Color online) (a) Single level model [Eqs. (9) and (10)] fitted to the experimentally measured conductance through a Deuterium molecule (Ref. 16). The parameters used for the fit are $\hbar \omega$ $=50 \mathrm{meV}, \tau=0.9825, \gamma_{\mathrm{eh}}=1.1 \times 10^{12} \mathrm{~s}^{-1}$, and $T=17 \mathrm{~K}$. (b) The ABL model [Eqs. (11) and (12)] fitted to the measured conductance through an atomic gold wire (experimental data from Ref. 4). The fit reveals the following parameters, $\hbar \omega=13.8 \mathrm{meV}, T=10 \mathrm{~K}, \gamma_{\mathrm{eh}}$ $=12 \times 10^{10} \mathrm{~s}^{-1}$, and $\gamma_{d}=3 \gamma_{\mathrm{eh}}$.

junction. We also note that both the size of the conductance step and the conductance slope (caused by heating) is fitted with only one parameter, the electron-hole damping rate $\gamma_{\mathrm{eh}}$.

The electronic structure of atomic gold chains are qualitatively different from the one level model. However, it is relatively straightforward to derive an alternating bond length (ABL) model. Inserting the electron-phonon matrix for an ABL phonon mode ${ }^{9}$ and using the Green's function for a half filled perfectly transmitting one-dimensional chain we obtain

$$
P_{\mathrm{ABL}}^{\mathrm{LOE}}=\gamma_{\mathrm{eh}} \hbar \omega\left[n_{B}(\hbar \omega)-n\right]+\frac{\gamma_{\mathrm{eh}}}{2} \frac{\pi \hbar}{\hbar \omega} \mathcal{P},
$$

$$
I_{\mathrm{ABL}}^{\mathrm{LOE}}=\frac{e^{2}}{\pi \hbar} V-\frac{e \gamma_{\mathrm{eh}}}{2} \frac{\pi \hbar}{e \hbar \omega} \mathcal{I}^{\mathrm{Sym}},
$$

where the only difference to the one-level model is that $\tau$ $=1$ (perfect transmission) and a factor of 2 caused by the absence of forward scattering from an ABL mode (the onelevel model has an equal amount of forward and back scattering). The ABL model is shown in Fig. 2, with the $\gamma_{\text {eh }}$ damping rate calculated directly from the DFT model. The main difference compared to the SCBA-LOE results is the assumption of perfect transmission through the chain. Fitting the ABL model to experimental data ${ }^{4}$ gives the very satisfactory fit shown in Fig. 3(b). We briefly note that the external damping $\gamma_{d}=3 \gamma_{\mathrm{eh}}$ is not negligible in contrast to the hydrogen case. In this paper we have used sharp phonon energies, cf. Eq. (2). However, if the phonon spectral function is known, it is possible to introduce broadening directly into Eqs. (3)-(7) from a finite phonon lifetime. 
We have derived simple and accurate approximations to describe the effect of phonon scattering on the conductance through nanoscale conductors. The approximate expressions greatly reduce the computational effort, compared to solving the SCBA equations. In addition, simple models were derived that provide insight and are suitable to fit experimental data.
The authors are grateful to D. Djukic, J. M. van Ruitenbeek, and N. Agraït for helpful discussions regarding their experimental work. This work, as part of the European Science Foundation EUROCORES Programme SASMEC, was supported by funds from the SNF and the EC 6th Framework Programme. Computational resources were provided by DCSC.
*Electronic address: mpn@mic.dtu.dk

${ }^{1}$ N. Agraït, A. L. Yeyati, and J. M. van Ruitenbeek, Phys. Rep. 377, 81 (2003).

${ }^{2}$ B. C. Stipe, M. A. Rezaei, and W. Ho, Science 280, 1732 (1998).

${ }^{3}$ R. H. M. Smit, Y. Noat, C. Untiedt, N. D. Lang, M. C. van Hemert, and J. M. van Ruitenbeek, Nature (London) 419, 906 (2002).

${ }^{4}$ N. Agraït, C. Untiedt, G. Rubio-Bollinger, and S. Vieira, Phys. Rev. Lett. 88, 216803 (2002).

${ }^{5}$ S. Braig and K. Flensberg, Phys. Rev. B 68, 205324 (2003).

${ }^{6}$ M. J. Montgomery, J. Hoekstra, T. N. Todorov, and A. P. Sutton, J. Phys.: Condens. Matter 15, 731 (2003).

${ }^{7}$ Y. Chen, M. Zwolak, and M. Di Ventra, Nano Lett. 4, 1709 (2004).

${ }^{8}$ M. Galperin, M. A. Ratner, and A. Nitzan, Nano Lett. 4, 1605 (2004).

${ }^{9}$ T. Frederiksen, M. Brandbyge, N. Lorente, and A.-P. Jauho, Phys. Rev. Lett. 93, 256601 (2004).

${ }^{10}$ T. Mii, S. G. Tikhodeev, and H. Ueba, Phys. Rev. B 68, 205406 (2003).

${ }^{11}$ B. N. J. Persson and M. Persson, Surf. Sci. 97, 609 (1980).
${ }^{12}$ H. Haug and A.-P. Jauho, Quantum Kinetics in Transport and Optics of Semiconductors (Springer-Verlag, Berlin, 1996).

${ }^{13}$ A. Pecchia and A. D. Carlo, Rep. Prog. Phys. 67, 1497 (2004).

${ }^{14}$ M. Head-Gordon and J. C. Tully, J. Chem. Phys. 96, 3938 (1992).

${ }^{15}$ J. K. Viljas, J. C. Cuevas, F. Pauly, and M. Häfner, during the review process a preprint [ cond-mat/0508470, (to be published)] using similar approximations appeared.

${ }^{16}$ D. Djukic, K. S. Thygesen, C. Untiedt, R. H. M. Smit, K. W. Jacobsen, and J. M. van Ruitenbeek, Phys. Rev. B 71, 161402(R) (2005).

${ }^{17}$ P. Hyldgaard, S. Hershfield, J. H. Davies, and J. W. Wilkins, Ann. Phys. (N.Y.) 236, 1 (1994).

${ }^{18}$ P. K. Hansma, Phys. Rep., Phys. Lett. 30, 145 (1977).

${ }^{19}$ The polaron term (Ref. 17) in the retarded self-energy in Eq. (2) has been neglected since it gives no "signal" at the phonon energy. However, it gives rise to two additional terms in the expression for the current [Eq. (5)] proportional to $V$ and $V^{2}$ and does not contribute to the power.

${ }^{20}$ For weak electron-phonon interaction, the division of power into the individual phonon modes is straightforward from Eq. (3). 